\title{
Design of $\Pi$ Impedance Matching Networks
}

\author{
Yichuang Sun J. K. Fidler \\ Department of Electronics, University of York \\ Heslington, York YO1 5DD, UK
}

\begin{abstract}
The $Q$-based method of $\Pi$ impedance matching network design is studied systematically. A more practical definition of the loaded quality factor $Q$ than that used hitherto is adopted. Designable conditions and design formulas based on the loaded $Q$ are analytically demonstrated. Accurate explicit expressions of network frequency responses and harmonic rejection in term of the loaded $Q$ are established and a method of determining the loaded $Q$ for the required harmonic attenuation is developed. We also formulate all tolerance and parasitic sensitivities and their relations to the loaded $Q$.
\end{abstract}

\section{INTRODUCTION}

Impedance matching networks are indispensible in radio transmitting and receiving systems and measuring circuits. The most widely used impedance matching network in practical situations is the low-pass $\Pi$ type due to its harmonic rejection capability and wider matchable impedance range [1-8]. Unlike $L$ networks whose element values are uniquely determined by the conjugate matching equations, there is one further degree of freedom in the accurate conjugate matching for II networks which allows us to select the loaded quality factor $Q$ to incorporate other requirements, for example harmonic rejection.

$Q$-based design theory of II impedance matching networks has undergone two periods of development. The first method was initiated by Pappenfus and Klippel in 1950 [1] and since then has been most widely used as a standard method for several decades. It was excellently outlined and developed by Grammer [2] and has appeared in various books and handbooks of electrical engineering [5, 6]. The second approach was first proposed by Gibson in 1969 [3] and has been further studied by Wingfield [4], which is highly commended in a recent book [6]. The two methods are mainly distinguished by the definition of the loaded $Q$ of the network. The first method treats $\omega C_{1} R_{1}$ as the loaded $Q$, while the second defines the loaded $Q$ as $\omega L / R_{B}$, where $R_{B}$ is the real part of $Z_{B}$, as shown in Fig.1.

This paper will further investigate the $Q$-based design theory of $\Pi$ impedance matching networks comprehensively. Many useful and interesting results will be presented.

\section{DESIGN FORMULAS AND DERIVATION}

Since in $Q$-based theory $Q$ is clearly the key design parameter, it is important to precisely decide what is the real loaded $Q$. Considering that resistance load transformation is often a good approximation of most practical cases and the basis of general complex impedance matching, discussion will be therefore carried out for resistance matching as shown in Fig.1. We denote $B_{C_{1}}=\omega C_{1}, B_{C_{2}}=\omega C_{2}, X_{L}=\omega L, Z_{A}=R_{A}-j X_{A}$ ( $Z_{A}$ is the Thevenin equivalent impedance), $Z_{B}=R_{B}-j X_{B}$

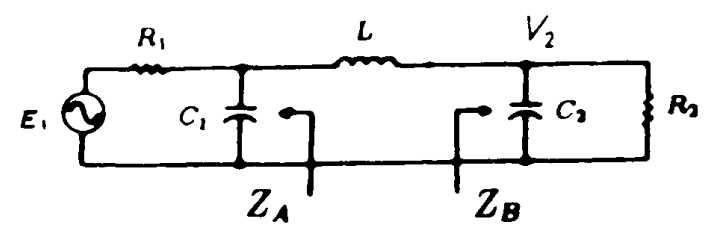

Figure 1: II impedance matching network

The loaded $Q$ is defined as $Q_{0}=X_{L} /\left(R_{A}+R_{B}\right)$. If we represent

$$
Q_{1}=R_{1} B_{C_{1}}, \quad Q_{2}=R_{2} B_{C_{2}}
$$

then according to the principle of series-parallel transformation we have $Q_{1}=X_{A} / R_{A}$, and $Q_{2}=X_{B} / R_{B}$.

On the other hand the conjugate matching conditions can be described as $R_{A}=R_{B}$, and $X_{L}=X_{A}+X_{B}$.

Thus the loaded $Q$ defined above can be formulated as

$$
Q_{0}=\left(Q_{1}+Q_{2}\right) / 2
$$

It should be pointed out that in the old standard formulas $Q_{1}$ is treated as the loaded $Q$ of the network $[1,2,5,6]$, and in the improved design formulas $X_{L} / R_{B}$ is used as the loaded $Q$ $[3,4,6]$. Apparently $Q_{1}$ is not the operating $Q$ of the network. $X_{L} / R_{B}$ is not either, since it does not consider the contribution of $R_{A}$ ( or $R_{1}$ ), although it was claimed $[3,4,6]$ to be more accurate than $Q_{1}$ used in the standard formulas.

To facilitate the design we derive that $R_{A}=R_{1} /\left(1+Q_{1}^{2}\right)$, $X_{A}=R_{1} Q_{1} /\left(1+Q_{1}^{2}\right), R_{B}=R_{2} /\left(1+Q_{2}^{2}\right), X_{B}=R_{2} Q_{2} /(1+$ $\left.Q_{2}^{2}\right)$. Substituting them into the conjugate matching conditions and with some manipulation we obtain

$$
\begin{gathered}
\left(1+Q_{1}^{2}\right) /\left(1+Q_{2}^{2}\right)=R_{1} / R_{2} \\
X_{L}=2 Q_{0} R_{1} /\left(1+Q_{1}^{2}\right)=2 Q_{0} R_{2} /\left(1+Q_{2}^{2}\right)
\end{gathered}
$$

Definition: Given $R_{1}, R_{2}$ and $Q_{0}$, the II network is said to be designable if and only if the values of elements $C_{1}, C_{2}$ and $L$ can be determined at any frequency. 
Theorem : The necessary and sufficient conditions of designability of the $\Pi$ network are

$$
\begin{array}{llll}
Q_{0} & \geq \frac{1}{2} \sqrt{\frac{R_{1}}{R_{2}}-1} & \text { for } & R_{1} \geq R_{2} \\
Q_{0} \geq \frac{1}{2} \sqrt{\frac{R_{2}}{R_{1}}-1} & \text { for } & R_{1} \leq R_{2}
\end{array}
$$

Equations $1-4$ constitute the basic design equations. From them we see that the designability of the network is equivalent to the existence of the non-negative solutions for $Q_{1}$ and $Q_{2}$ of equations 2 and 3. Equation 3 may be written as $Q_{2}^{2}=$ $\frac{R_{2}}{R_{2}}\left(1+Q_{1}^{2}\right)-1$. When $R_{1} \geq R_{2}$, to ensure that $Q_{2} \geq 0$ we must have $Q_{1} \geq \sqrt{R_{1} / R_{2}-1}$. This can also be expressed in term of $Q_{0}$ by using equation 2 as $Q_{0} \geq \frac{1}{2} \sqrt{R_{1} / R_{2}-1}$. Similarly, when $R_{1} \leq R_{2}$, we must have $Q_{2} \geq \sqrt{R_{2} / R_{1}-1}$ to guarantee that $Q_{1} \geq 0$. Or in term of $Q_{0}, Q_{0} \geq \frac{1}{2} \sqrt{R_{2} / R_{1}-1}$ must be satisfied.

On the other hand if condition 5 or 6 holds, solving equations 2 and 3 yields non-negative $Q_{1}$ and $Q_{2}$, given by

$$
\begin{aligned}
& Q_{1}=\left(2 Q_{0} R_{1}-\sqrt{4 Q_{0}^{2} R_{1} R_{2}-\left(R_{1}-R_{2}\right)^{2}}\right) /\left(R_{1}-R_{2}\right) \\
& Q_{2}=\left(2 Q_{0} R_{2}-\sqrt{4 Q_{0}^{2} R_{1} R_{2}-\left(R_{1}-R_{2}\right)^{2}}\right) /\left(R_{2}-R_{1}\right)
\end{aligned}
$$

In particular, when $R_{1}=R_{2}$, condition 5 or 6 becomes $Q_{0} \geq 0$ and equations 7 and 8 reduce to

$$
Q_{1}=Q_{2}=Q_{0}
$$

Now the Q-based design procedure may be summarized as: a. Specify $R_{1}, R_{2}$ and $Q_{0}$. b. Verify the designable conditions 5 and 6 (if they are not met, redesignate $Q_{0}$ until they are satisfied), c. Compute $Q_{1}$ and $Q_{2}$ using equations 7-9. d. Determine $X_{L}, B_{C_{1}}$ and $B_{C_{2}}$ from equations 1 and 4 .e. Calculate $L, C_{1}$ and $C_{2}$ at any given frequency.

For some special cases design equations may be simplified. When $R_{1}=R_{2}=R$, the design formulas become

$$
B_{C_{1}}=B_{C_{2}}=Q_{0} / R, \quad X_{L}=2 Q_{0} R /\left(1+Q_{0}^{2}\right)
$$

The designed $\Pi$ network is therefore symmetrical.

Simplified design formulas for $R_{1} \gg R_{2}$ and $R_{1} \ll R_{2}$, are respectively given by

$$
\begin{gathered}
B_{C_{1}}=2 Q_{0} / R_{1}, \quad X_{L}=2 Q_{0} R_{1} /\left(1+4 Q_{0}^{2}\right) \\
B_{C_{2}}=\frac{1}{R_{2}} \sqrt{\frac{R_{2}}{R_{1}}\left(1+4 Q_{0}^{2}\right)-1}
\end{gathered}
$$

and

$$
\begin{gathered}
B_{C_{2}}=2 Q_{0} / R_{2}, \quad X_{L}=2 Q_{0} R_{2} /\left(1+4 Q_{0}^{2}\right) \\
B_{C_{1}}=\frac{1}{R_{1}} \sqrt{\frac{R_{1}}{R_{2}}\left(1+4 Q_{0}^{2}\right)-1}
\end{gathered}
$$

From equations 2 and 3 we can see that if $R_{1} \gg R_{2}, Q_{1} \gg$ $Q_{2}$ and $Q_{1} \approx 2 Q_{0}$; if $R_{1} \ll R_{2}, Q_{1} \ll Q_{2}$ and $Q_{2} \approx 2 Q_{0}$. For both cases we can further calculate $Q_{2}$ and $Q_{1}$ by substituting the approximate expressions of $Q_{1}$ and $Q_{2}$ into equation 3, respectively. Then using equations 1 and 4 we can get the above formulas.
From the designable conditions we can also see that given $R_{1}$ and $R_{2}$, the minimum loaded $Q_{0}$, denoted by $Q_{0 \text { min }}$ is determined by

$$
Q_{0 \min }=\frac{1}{2} \sqrt{\frac{R_{1}}{R_{2}}-1} \quad \text { or } \quad=\frac{1}{2} \sqrt{\frac{R_{2}}{R_{1}}-1}
$$

for $R_{1}>R_{2}$ and $R_{1}<R_{2}$, respectively.

If $Q_{0}=Q_{0 \min }$, the $\Pi$ network reduces to $L$ networks. For example, for $R_{1}>R_{2}$ there is $Q_{0}=Q_{0 \text { min }} \Longleftrightarrow Q_{1}=$ $2 Q_{0 \text { min }} \Longleftrightarrow Q_{2}=0$. Thus we can attain

$$
B_{C_{2}}=0, \quad B_{C_{1}}=\frac{1}{R_{1}} \sqrt{\frac{R_{1}}{R_{2}}-1}, \quad X_{L}=R_{2} \sqrt{\frac{R_{1}}{R_{2}}-1}
$$

In a similar way, for $R_{1}<R_{2}$ we have $Q_{0}=Q_{0 \min } \Longleftrightarrow$ $Q_{1}=0 \Longleftrightarrow Q_{2}=2 Q_{0 \mathrm{~min}}$, which leads to

$$
B_{C_{1}}=0, \quad B_{C_{2}}=\frac{1}{R_{2}} \sqrt{\frac{R_{2}}{R_{1}}-1}, \quad X_{L}=R_{1} \sqrt{\frac{R_{2}}{R_{1}}-1}
$$

From the above discussion we can see that the design of $\mathrm{L}$ networks is a special case of that of the II network. What interests us is that it is a limiting example. As will be seen later many interesting results relate to this limit.

It is noted that combining the designable conditions gives

$$
1 /\left(1+4 Q_{0}^{2}\right) \leq R_{1} / R_{2} \leq 1+4 Q_{0}^{2}
$$

Thus given $Q_{0}$ and $R_{2}$, the bound of $R_{1}$, and given $Q_{0}$ and $R_{1}$, the range of $R_{2}$, and finally given $Q_{0}$, the bound of the ratio $k=R_{1} / R_{2}$ all can be determined from this equation.

These three cases are very practical. For example, the impedance matching network may be used to match the output resistance of a $\mathrm{rf}$ power amplifier to the characteristic impedance of transmission lines, with the former being usually variable and the latter being for instance 50ohm [1-8]. In the design of the antenna tuning unit, on the other hand, the matching network is required to transform the changeable antenna impedance to the $50 \mathrm{ohm}$ transmission line characteristic impedance [5-8]. In some general interstage coupling problems the two impedances to be matched both may be changeable. For all these practical situations equation 10 gives the allowable changing ranges within which when the related $R_{1}, R_{2}$ or $k$ varies, conjugate match can be achieved. It is clear that if one wants to match all the allowable range for respective cases, one will need adjustable elements. In this end equation 10 may also be used to determine the corresponding tunable ranges of element values.

If the $\Pi$ network is desired to operate over a band of frequencies $f_{\min } \leq f \leq f_{\max }$ for given $R_{1}, R_{2}$ and $Q_{0}$, the ranges of $C_{1}, C_{2}$ and $L$ to cover the frequency band may be determined in two steps: First utilize the basic design procedure to get $B_{C_{1}}, B_{C_{2}}$ and $X_{L}$ for the given $R_{1}, R_{2}$ and $Q_{0}$. Then determine the ranges of $C_{1}, C_{2}$ and $L$, for example $B_{C_{2}} /\left(2 \pi f_{\max }\right) \leq C_{2} \leq B_{C_{2}} /\left(2 \pi f_{\min }\right)$.

As discussed previously, as long as $R_{1}, R_{2}$ and $Q_{0}$ are given $B_{C_{1}}, B_{C_{2}}$ and $X_{L}$ are accordingly fixed. However, the real loaded $Q_{0}$ of the network is dependent on the operating frequency. In order to keep $Q_{0}$ constant as given when the frequency changes, we must therefore adjust the values of $C_{1}, C_{2}$ 
and $L$ to guarantee that $B_{C_{1}}, B_{C_{2}}$ and $X_{L}$ are not altered. Thus a uniform or consistent performance of the network can be achieved at different frequencies within the specified range.

\section{FREQUENCY RESPONSES AND $Q_{0}$ SELECTION}

Defining $H(s)=V_{2} / E_{1}$, simple circuit analysis yields

$$
\begin{gathered}
H(s)=R_{2} /\left[s^{3} L C_{1} C_{2} R_{1} R_{2}+s^{2}\left(L C_{1} R_{1}+L C_{2} R_{2}\right)\right. \\
\left.+s\left(L+C_{1} R_{1} R_{2}+C_{2} R_{1} R_{2}\right)+\left(R_{1}+R_{2}\right)\right]
\end{gathered}
$$

By substituting $s=j \omega$ we can attain the complex frequency response $H(j \omega)=|H(j \omega)| e^{j \Psi(\omega)}$. With $k=R_{1} / R_{2}$ and $\omega_{m}$ denoting the matching angular frequency and using the design formulas obtained we can derive the magnitude and the phase frequency responses:

$$
\begin{gathered}
|H(j \omega)|=2\left[(k+1) Q_{0}-\sqrt{4 k Q_{0}^{2}-(k-1)^{2}}\right] /\left\{\left[2(k+1)^{2} Q_{0}-\right.\right. \\
\left.2(k+1) \sqrt{4 k Q_{0}^{2}-(k-1)^{2}}-2(k-1)^{2} Q_{0}\left(\frac{\omega}{\omega_{m}}\right)^{2}\right]^{2}+\left[\left(3(k-1)^{2}\right.\right. \\
\left.-8 k Q_{0}^{2}+2(k+1) Q_{0} \sqrt{4 k Q_{0}^{2}-(k-1)^{2}}\right)\left(\frac{\omega}{\omega_{m}}\right)+ \\
\left.\left.\left(8 k Q_{0}^{2}-2(k+1) Q_{0} \sqrt{4 k Q_{0}^{2}-(k-1)^{2}}-(k-1)^{2}\right)\left(\frac{\omega}{\omega_{m}}\right)^{3}\right]^{2}\right\}^{\frac{1}{2}}
\end{gathered}
$$$$
\Psi(\omega)=-\arctan \left(\left\{\left[3(k-1)^{2}-8 k Q_{0}^{2}+\right.\right.\right.
$$$$
\left.2(k+1) Q_{0} \sqrt{4 k Q_{0}^{2}-(k-1)^{2}}\right] \frac{\omega}{\omega_{m}}+
$$$$
\left.\left[8 k Q_{0}^{2}-2(k+1) Q_{0} \sqrt{4 k Q_{0}^{2}-(k-1)^{2}}-(k-1)^{2}\right]\left(\frac{\omega}{\omega_{m}}\right)^{3}\right\} /
$$$$
\left.\left[2(k+1)^{2} Q_{0}-2(k+1) \sqrt{4 k Q_{0}^{2}-(k-1)^{2}}-2(k-1)^{2} Q_{0}\left(\frac{\omega}{\omega_{n}}\right)^{2}\right]\right)
$$

The magnitude and phase at $\omega_{m}$ are derived as, respectively $\left|H\left(j \omega_{m}\right)\right|=1 /(2 \sqrt{k})$ and

$$
\Psi\left(\omega_{m}\right)=-\arctan \left(\frac{(k-1)^{2}}{4 k Q_{0}-(k+1) \sqrt{4 k Q_{0}^{2}-(k-1)^{2}}}\right)
$$

We can see that the network frequency characteristics are completely determined by $Q_{0}$ and $k$. For the known resistances $R_{1}$ and $R_{2}$ (that is, $k$ ) and $Q_{0}$ using these explicit formulas we can readily analyse the network frequency performance.

In the design of the $\Pi$ matching network the selection of the loaded $Q$ is of prime importance. It is known that in the selection of the loaded $Q$, the most basic and important consideration is to meet the designable condition, that is, $Q_{0} \geq Q_{0 \mathrm{~min}}$. The $Q_{0}$ specified by all other requirements must comply with this restriction. On the other hand, the $\Pi$ network is most often the choice mainly because it can provide the expected harmonic suppression apart from the wider matchable impedance range. The harmonic rejection requirement must thus be taken into consideration when choosing $Q_{0}$.

The selectivity of a network may be defined as $S(\omega)=$ $|H(j \omega)| /\left|H\left(j \omega_{m}\right)\right|$. For the II network it can be expressed as (Note that $Q_{0}$ is highlighted as a variable)

$$
\begin{gathered}
S\left(\omega, Q_{0}\right)=4 \sqrt{k}\left[(k+1) Q_{0}-\sqrt{4 k Q_{0}^{2}-(k-1)^{2}}\right] / \\
\left\{\left[\left(2(k+1)^{2}-2\left(\frac{\omega}{\omega_{m}}\right)^{2}(k-1)^{2}\right) Q_{0}\right.\right.
\end{gathered}
$$

$$
\begin{gathered}
\left.-2(k+1) \sqrt{4 k Q_{0}^{2}-(k-1)^{2}}\right]^{2}+ \\
{\left[\left(3\left(\frac{\omega}{\omega_{m}}\right)-\left(\frac{\omega}{\omega_{m}}\right)^{3}\right)(k-1)^{2}-8\left(\frac{\omega}{\omega_{m}}-\left(\frac{\omega}{\omega_{m}}\right)^{3}\right) k Q_{0}^{2}\right.} \\
\left.\left.+2\left(\frac{\omega}{\omega_{m}}-\left(\frac{\omega}{\omega_{m}}\right)^{3}\right)(k+1) Q_{0} \sqrt{4 k Q_{0}^{2}-(k-1)^{2}}\right]^{2}\right\}^{\frac{1}{2}}
\end{gathered}
$$

For the given $R_{1}$ and $R_{2}$ to be matched and the requirement of harmonic rejection, the determination of $Q_{0}$ can be easily done by using this expression since any order harmonic performance in term of $Q_{0}$ with $k$ being as the parametric variable can be obtained. For instance, the second harmonic rejection is formulated as

$$
\begin{gathered}
S\left(2 \omega_{m}, Q_{0}\right)=2 \sqrt{k}\left[(k+1) Q_{0}-\sqrt{4 k Q_{0}^{2}-(k-1)^{2}}\right] / \\
\left\{\left[\left((k+1)^{2}-4(k-1)^{2}\right) Q_{0}-(k+1) \sqrt{4 k Q_{0}^{2}-(k-1)^{2}}\right]^{2}+\right. \\
\left.\left[24 k Q_{0}^{2}-6(k+1) Q_{0} \sqrt{4 k Q_{0}^{2}-(k-1)^{2}}-(k-1)^{2}\right]^{2}\right\}^{\frac{1}{2}}
\end{gathered}
$$

and the third harmonic attenuation is given by

$$
\begin{gathered}
S\left(3 \omega_{m}, Q_{0}\right)=2 \sqrt{k}\left[(k+1) Q_{0}-\sqrt{4 k Q_{0}^{2}-(k-1)^{2}}\right] / \\
\left\{\left[\left((k+1)^{2}-18(k-1)^{2}\right) Q_{0}-(k+1) \sqrt{4 k Q_{0}^{2}-(k-1)^{2}}\right]^{2}+\right. \\
\left.\left[96 k Q_{0}^{2}-24(k+1) Q_{0} \sqrt{4 k Q_{0}^{2}-(k-1)^{2}}-9(k-1)^{2}\right\}^{2}\right\}^{\frac{1}{2}}
\end{gathered}
$$

As an illustration, with $k=0.0625$ the second harmonic attenuation in $d B$ is poltted with respect to $Q_{0}$ in Fig.2.

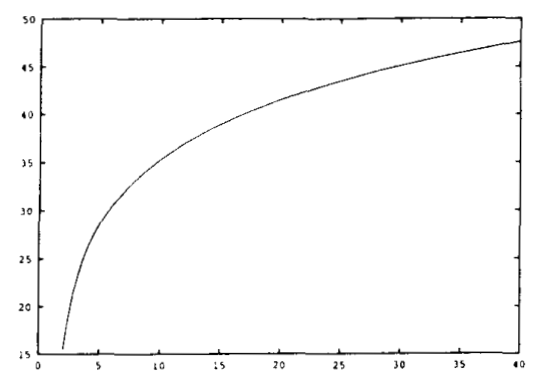

Figure 2: Second harmonic and $Q_{0}$

It can be seen that for the given $k$ and the required harmonic depression the corresponding $Q_{0}$ can be determined graphically. For example, when $R_{1}=50 \mathrm{ohms}$ and $R_{2}=800 \mathrm{ohms}$ and $35 \mathrm{~dB}$ second harmonic suppression are expected we can determine $Q_{0}=10$ from the graph in Fig. 2. In the case that the minimum allowable harmonic rejection is specified, we may first find the corresponding $Q_{0}$, denoted by $Q_{0 L}$, and then arbitrarily select $Q_{0}$ which meets $Q_{0} \geq Q_{0 L}$. If both second and third harmonic rejections are specified, we can choose $Q_{0}=\max \left\{Q_{0 s e c}, Q_{0 t h i}\right\}$, where $Q_{0 \text { sec }}$ and $Q_{0 t h i}$ correspond to the second and the third harmonic requirements respectively. It is clear that $\Pi$ networks have higher harmonic suppression than $\mathrm{L}$ networks since the $\mathrm{L}$ networks correspond to $Q_{0 \mathrm{~min}}$.

\section{SENSITIVITY ANALYSIS}

Sensitivity is an important criterion for assessing network quality. The magnitude and phase tolerance sensitivities are respectively defined as

$$
T S_{t}^{|H(j \omega)|}=\frac{t}{|H(j \omega)|} \frac{\partial|H(j \omega)|}{\partial t} \text { and } T S_{t}^{\Psi(\omega)}=t \frac{\partial \Psi(\omega)}{\partial t}
$$


where $t$ represents any normal circuit parameter.

According to the definitions the tolerance sensitivities of the magnitude to $L, C_{1}, C_{2}, R_{1}$ and $R_{2}$ at $\omega_{m}$ and at the nominal state can be directly formulated as $0,0,0,-0.5$, and 0.5 respectively, and the phase tolerance sensitivities to them are derived as $-Q_{0},-Q_{1} / 2,-Q_{2} / 2,0$, and 0 consecutively.

In the above we assumed that the $\Pi$ network is lossless. However, in practical situations reactive components have always some parasitic losses which can be equivalent to a parallel conductance for a capacitor and a series resistance for an inductor. The magnitude and phase sensitivities to such parasitics are denoted by

$$
P S_{p}^{|H(j \omega)|}=\frac{1}{|H(j \omega)|} \frac{\partial|H(j \omega)|}{\partial p} \text { and } P S_{p}^{\Psi(\omega)}=\frac{\partial \Psi(\omega)}{\partial p}
$$

in which $p$ may be the loss conductance $g_{C_{i}}$ of capacitor $C_{i}$ $i=1,2$, or the loss resistance $r_{L}$ of inductor $L$.

They can be respectively computed from the phase and magnitude tolerance sensitivities to the associated reactive elements based on the Cauchy-Riemann theorem, which states, for example $P S_{r_{L}}^{|H(j \omega)|}=\frac{1}{\omega L} T S_{L}^{\psi(\omega)}$ and $P S_{g_{C_{2}}}^{\psi(\omega)}=$ $-\frac{1}{\omega C_{2}} T S_{C_{2}}^{|H(j \omega)|}$. Thus we can easily demonstrate that

$$
P S_{r_{L}}^{\left|H\left(j \omega_{m}\right)\right|}=-\frac{2 k Q_{0}\left[(k+1) Q_{0}-\sqrt{4 k Q_{0}^{2}-(k-1)^{2}}\right]}{R_{1}(k-1)^{2}}
$$

and $P S_{g_{C}}^{\left|H\left(j \omega_{m}\right)\right|}=-R_{i} / 2, i=1,2$, and that all phase parasitic sensitivities are zero.

The above analysis reveals that the $\Pi$ matching network has very low sensitivities. Clearly all magnitude tolerance sensitivities, the phase tolerance sensitivities to $R_{1}$ and $R_{2}$ and all phase parasitic sensitivities reach the minimum.

However the phase tolerance sensitjities to $L, C_{1}$ and $C_{2}$ are proportional to $Q_{0}, Q_{1}$ and $Q_{2}$, respectively. Therefore they will become higher as $Q_{0}$ increases. Obviously the minimum sensitivities correspond to $Q_{0}=Q_{0 \text { min }}$ and they are $T S_{L}^{\Psi\left(\omega_{m}\right)}=T S_{C_{1}}^{\Psi\left(\omega_{m}\right)}=-\frac{1}{2} \sqrt{k-1}, T S_{C_{2}}^{\Psi\left(\omega_{m}\right)}=0$, for $k>1$ and $T S_{L}^{\Psi\left(\omega_{m}\right)}=T S_{C_{2}}^{\Psi\left(\omega_{m}\right)}=-\frac{1}{2} \sqrt{\frac{1}{k}-1}, T S_{C_{1}}^{\Psi\left(\omega_{m}\right)}=0$, for $k<1$. It is of interest to note that in these minimum sensitivity cases the sensitivity to the inductance is equal to that to the corresponding capacitance and the $\Pi$ network reduces to the respective $L$ type. Generally we can conclude that the II network has larger phase tolerance sensitivities to reactive elements than the $L$ counterpart.

As for the magnitude parasitic sensitivities, we distinguish two cases. In the first case we see that the sensitivities to each capacitance loss are directly proportional to the termination resistance on the side of the capacitor. They can be high when $R_{1}$ and/or $R_{2}$ are large and they are fixed for the given $R_{1}$ and $R_{2}$. These indicate respectively that it may not be safe to blindly ignore the effects of capacitor loss in some situations and that there is no way to decrease these sensitivities for the given matching problem. Fortunately the parasitic loss conductances of capacitors are normally small.

In the second the sensitivity to the inductor loss $\left(r_{L}\right)$ is a function of $Q_{0}$ when $k$ is specified. This sensitivity deserves a special attention because inductor loss is usually larger compared with capacitor loss. Differentiating the sensitivity function (equation 11) with respect to $Q_{0}$ and letting the result be zero we can solve that $Q_{0}=\frac{1}{2} \sqrt{k-1}$ for $k>1$ and $Q_{0}=\frac{1}{2} \sqrt{\frac{1}{k}-1}$ for $k<1$. Substituting them into the sensitivity function we attain the minimum sensitivities to $r_{L}$ for $k>1$ and $k<1$ as $-1 /\left(2 R_{2}\right)$ and $-1 /\left(2 R_{1}\right)$ respectively. Interestingly the minimum sensitivity to $r_{L}$ is inversely proportional to the smaller of the two termination resistances and it corresponds to $L$ networks since the $Q_{0}$ resulting in the minimum sensitivity is the same as the minimum $Q_{0}$ required by the network designability.

As a summary when $Q_{0}=Q_{0 \min }$ the II network degenerates to $\mathrm{L}$ networks, has the minimum phase tolerance sensitivities to the normal reactive elements $L, C_{1}$ and $C_{2}$ and the minimum magnitude parasitic sensitivity to the inductor loss resistance $r_{L}$. However the harmonic suppression meanwhile reaches the worst. Generally when $Q_{0}$ increases the harmonic rejection increases and the relevent sensitivities become larger. Therefore a trade-off between harmonic attenuation and sensitivities (especially that to the inductor loss ) may need to be made in the real design.

\section{CONCLUSIONS}

A $Q$-based design theory of impedance matching networks has been explored. The $Q$-based method proposed is very attractive due to extremely simple algebraic design formulas, the capability of achieving both precise impedance matching and harmonic suppression, and the adoption of the well-known $Q$ concept. Although the method has been discussed for resistance matching of the $\Pi$ network, the results obtained are dually suitable for $T$ networks which are also extensively used and can be applied to general complex impedance matching problems with a little improvement. Furthermore, they may also be extended to the design of more complicated matching networks which use $L, \Pi$ or $T$ networks as basic sections. Some of the problems are dealt with in [8] and others will be the subject of a further publication.

\section{REFERENCES}

[1] E. W. Pappenfus and K. L. Klippel, pi network tank circuits, $C Q$, September, p.27, 1950

[2] G. Grammer, Simplified design of impedance-matching networks, part 1, 2 and 3, March pp 38-42, April pp 32-35 and May pp 29-34, QST, 1957.

[3] H. L. Gibson, An improved design method for $\mathrm{Pj}$ and $\mathrm{L} \mathrm{Pi}$ network couplers, Radio Communication, pp 390-392, 1969.

[4] E. A. Wingfield, New and improved formulas for the design of Pi and Pi-L networks, QST, August, pp 23-29, 1983.

[5] C. Bowick, $R F$ circuit design, Howard W. Sams \& Co., Inc., Indianapolis, 1982.

[6] M. W. Maxwell, Reflections: Transmission lines and antennas, American Radio Relay League, 1990.

[7] J. K. Fidler and Y. Sun. Computer-aided determination of impedance matching domain, IEE 12th Saraga Colloquium on Electronic Filters, 1992.

[8] Y. Sun and J. K. Fidler. Practical considerations of impedance matching network design, Proc. IEE Sixth Int. Conf. HF Radio Systems and Techniques, 1994. 